\title{
Unsteady Boundary-Layer Flow over Jerked Plate Moving in a Free Stream of Viscoelastic Fluid
}

\author{
Sufian Munawar, ${ }^{1,2}$ Ahmer Mehmood, ${ }^{3}$ Asif Ali,, ${ }^{1,4}$ and Najma Saleem ${ }^{1}$ \\ ${ }^{1}$ Department of Mathematics and Basic Sciences, Prince Mohammad Bin Fahd University, Al Khobar 31952, Saudi Arabia \\ ${ }^{2}$ Department of Informatics and Systems, University of Management and Technology, Lahore 54000, Pakistan \\ ${ }^{3}$ Department of Mathematics (FBAS), International Islamic University Islamabad, Islamabad 44000, Pakistan \\ ${ }^{4}$ Department of Mathematics, Quaid-i-Azam University, Islamabad 44000, Pakistan
}

Correspondence should be addressed to Sufian Munawar; sufian.munawar@hotmail.com

Received 10 February 2014; Accepted 11 April 2014; Published 6 May 2014

Academic Editor: Tiegang Fang

Copyright (c) 2014 Sufian Munawar et al. This is an open access article distributed under the Creative Commons Attribution License, which permits unrestricted use, distribution, and reproduction in any medium, provided the original work is properly cited.

\begin{abstract}
This study aims to investigate the unsteady boundary-layer flow of a viscoelastic non-Newtonian fluid over a flat surface. The plate is suddenly jerked to move with uniform velocity in a uniform stream of non-Newtonian fluid. Purely analytic solution to governing nonlinear equation is obtained. The solution is highly accurate and valid for all values of the dimensionless time $0 \leq \tau<\infty$. Flow properties of the viscoelastic fluid are discussed through graphs.
\end{abstract}

\section{Introduction}

Boundary-layer flows over flat surfaces are fundamental for the understanding of aerodynamical properties of the flow such as wall friction and the dynamical drag. Due to the boundary-layer theory proposed by Prandtl in 1904 [1], it became possible to calculate the drag on a sphere accurately. Blasius [2] investigated the boundary-layer flow past a flat plate at zero incidence. The wall friction in Blasius flow was calculated to be 0.332057 approximately. The same twodimensional boundary-layer flow was studied by Sakiadis [3] over a moving wall in a still fluid. Sakiadis reported the value of skin friction at the wall to be $0.444 \ldots$ for his case. Sakiadis also predicted that the drag force for his flow problem was $34 \%$ greater than the drag force for the Blasius flow [2]. The theoretical results of Sakiadis [3] were experimentally confirmed by Tsuo et al. [4]. In both studies [2,3], either fluid or plate is assumed to be at rest. However, the most practical situation might be the one in which both the fluid and the plate are moving. Such a flow situation is more practical in studying the aerodynamical properties of the flow. Keeping this fact in mind, Klemp and Acrivos [5] considered twodimensional boundary-layer flow of a viscous fluid over a flat surface moving in a stream of constant velocity. Hussaini et al. [6] determined similarity solution of the boundary layer equation with upstream moving wall. Later on in 2003, Fang $[7,8]$ studied the similarity solution of boundary-layer flow and heat transfer for steady case. In [7, 8], Fang studied both cases, namely, when the plate is moving in the direction of free stream or in opposite direction to the free stream. Recently, Mehmood et al. $[9,10]$ considered unsteady boundary-layer flow over an impulsively started moving plate in a free stream with parallel and antiparallel motion.

Boundary-layer flows over moving surfaces find important industrial applications such as in the manufacturing of food and paper, plastic sheet extrusion, application of coating of paints layers on surfaces, and many other activities (see for instance [11, 12]). In such industrial applications, the fluid is observed to have non-Newtonian character. Due to these important industrial applications, the study of nonNewtonian fluid flows over moving surface needs attention. In 1969, Fox et al. [13] investigated the non-Newtonian flow over a moving surface using the power-law model. The flow of a power-law fluid over a moving plate in a parallel free stream was investigated by Hassanien [14]. Further, Hassanien [15] investigated the boundary-layer flow over a moving plate in a free stream of second-grade fluid with heat transfer analysis.

In all the abovementioned studies for non-Newtonian fluids, the authors considered steady flow. However, there are many practical applications in which the flow is essentially 
unsteady. For unsteady problems governed by nonlinear equations, it is very difficult to obtain an analytic solution valid for all time. Perturbation methods are sometimes used to get analytic solution valid for small time [16-18]. Currently, Liao [19, 20], Xu et al. [21], Cheng et al. [22], Wang [23], Abbasbandy et al. [24], Xu and Liao [25], Mehmood et al. [9, $10,26,27$ ], and Munawar et al. [28] obtained purely analytic solution for unsteady flows by homotopy analysis method [29]. In $[9,10,19-28]$, the authors have shown their results to be valid uniformly for all time. Homotopy analysis method is a powerful analytic technique (introduced by Liao [29]) for highly nonlinear problems. The technique has been widely used by a number of researchers in nonlinear problems arising in science and engineering [30-41].

In the present study, we extend the work of Mehmood and Ali [9] for the non-Newtonian case. The fluid considered is of second-grade type and the problem is solved by homotopy analysis method. The objective of this study is twofold: firstly to report a purely analytic solution to the considered unsteady problem and secondly to investigate the viscoelastic effects on the flow characteristics. The solution is highly accurate and is uniformly valid for all time in the whole spatial domain. The accuracy and convergence of present analytic solution are discussed in detail. The outlines of the paper are as follows.

Section 2 contains the mathematical formulation and the HAM solution of the problem. The issue of convergence and accuracy of HAM results is also discussed in Section 2. Section 3 consists of graphical representation of results and their discussion. Section 4 is reserved for concluding remarks.

\section{Formulation of the Problem}

Consider the unsteady incompressible flow of second-grade fluid over an infinite plate at $y=0$. The fluid occupies the half space $y>0$ and at infinity it is assumed that fluid is flowing with the constant free-stream velocity $U$. Initially, fluid is at rest; then at time $t \geq 0$, suddenly plate starts its motion with the constant velocity $\lambda U$, where $\lambda$ is the ratio of plate velocity to the free-stream velocity. For an incompressible homogeneous fluid of second-grade type, the Cauchy stress tensor $\mathbf{T}$ is related to the deformation field through

$$
\mathbf{T}=-p \mathbf{I}+\mu \mathbf{A}_{1}+\alpha_{1} \mathbf{A}_{2}+\alpha_{2} \mathbf{A}_{1}^{2}
$$

where $p$ is the pressure, $\mathbf{I}$ is the identity tensor, $\mu$ is the dynamic viscosity, $\alpha_{1}$ and $\alpha_{2}$ are the viscoelastic parameters, and the kinematical tensors $\mathbf{A}_{1}$ and $\mathbf{A}_{2}$ are given by

$$
\begin{gathered}
\mathbf{A}_{1}=\mathbf{L}+\mathbf{L}^{T}, \\
\mathbf{A}_{2}=\frac{d \mathbf{A}_{1}}{d t}+\mathbf{A}_{1} \mathbf{L}+\mathbf{L}^{T} \mathbf{A}_{1},
\end{gathered}
$$

where $d / d t$ is the material time derivative and $\mathbf{L}$ is velocity gradient. This rheological model was first introduced by Rivlin and Ericksen [42].
Experimental data available for the large number of viscoelastic fluids suggests that, in order to satisfy the thermodynamical analysis, some restrictions must be put on the signs and magnitudes of the material parameters [43]

$$
\mu \geq 0, \quad \alpha_{1} \geq 0, \quad \alpha_{1}+\alpha_{2}=0 .
$$

Under the above assumptions and conditions, the boundary layer equations governing the unsteady laminar flow of an incompressible viscoelastic fluid due to an impulsive motion of plate are given by

$$
\begin{gathered}
\frac{\partial u}{\partial x}+\frac{\partial v}{\partial y}=0 \\
\frac{\partial u}{\partial t}+u \frac{\partial u}{\partial x}+v \frac{\partial u}{\partial y}=\frac{\partial T_{x x}}{\partial x}+\frac{\partial T_{x y}}{\partial x}
\end{gathered}
$$

where $T_{x x}$ and $T_{x y}$ are the components of the stress tensor given by

$$
\begin{gathered}
T_{x x}=-p+2 \mu \frac{\partial u}{\partial x}+\alpha_{1}\left[2 \frac{\partial^{2} u}{\partial t \partial y}+2 u \frac{\partial^{2} u}{\partial x^{2}}\right. \\
\left.+2 v \frac{\partial^{2} u}{\partial x \partial y}+\left(\frac{\partial v}{\partial x}\right)^{2}-\left(\frac{\partial u}{\partial y}\right)^{2}\right] \\
T_{x y}=\mu\left(\frac{\partial u}{\partial y}\right)+\alpha_{1}\left[\frac{\partial^{2} u}{\partial t \partial y}+u \frac{\partial^{2} u}{\partial x \partial y}\right. \\
\left.+v \frac{\partial^{2} u}{\partial y^{2}}+2 \frac{\partial u}{\partial x} \frac{\partial u}{\partial y}\right]
\end{gathered}
$$

Using (6) and (7) in (5) and assuming the constant pressure, we have

$$
\begin{aligned}
\frac{\partial u}{\partial t} & +u \frac{\partial u}{\partial x}+v \frac{\partial u}{\partial y} \\
& =v \frac{\partial^{2} u}{\partial y^{2}}+\frac{\alpha_{1}}{\rho}\left[\frac{\partial^{2} u}{\partial t \partial y^{2}}+\frac{\partial}{\partial x}\left(u \frac{\partial^{2} u}{\partial y^{2}}\right)+v \frac{\partial^{3} v}{\partial y^{3}}+\frac{\partial u}{\partial y} \frac{\partial^{2} v}{\partial y^{2}}\right]
\end{aligned}
$$

subject to the boundary conditions (when $t \geq 0$ )

$$
\begin{aligned}
& u=\lambda U, \quad v=0, \quad \text { at } y=0, \\
& u \longrightarrow U, \quad \text { as } y \longrightarrow \infty,
\end{aligned}
$$

in which $\nu$ is the kinematic viscosity, $\rho$ is the fluid density, and $u(x, y, t)$ and $v(x, y, t)$ are the velocity components in the $x$ - and $y$-directions, respectively. The initial condition (when $t<0)$ is given by

$$
u=v=0, \quad \forall x, y .
$$

Introducing the similarity transformations [9]

$$
\begin{array}{ll}
\eta=\sqrt{\frac{U}{\nu x \xi}} y, & \psi=\sqrt{\nu U x \xi} f(\eta, \xi), \\
\xi=1-e^{-\tau}, & \tau=\frac{U}{x} t,
\end{array}
$$


the governing equation (8) readily transforms to

$$
\begin{array}{r}
{[\xi-\alpha(1-\xi)] f^{\prime \prime \prime}-\xi^{2}(1-\xi) \frac{\partial f^{\prime}}{\partial \xi}+\frac{1}{2} \eta \xi(1-\xi) f^{\prime \prime}} \\
+\frac{1}{2} \xi^{2} f f^{\prime \prime}=-\alpha\left[\xi(1-\xi) \frac{\partial f^{\prime \prime \prime}}{\partial \xi}-\frac{\eta}{2}(1-\xi) f^{i v}\right. \\
\left.+\frac{1}{2} \xi f^{\prime \prime 2}-\frac{1}{2} \xi f f^{i v}-\xi f^{\prime} f^{\prime \prime \prime}\right],
\end{array}
$$

with boundary conditions

$$
f(0, \xi)=0,\left.\quad \frac{\partial f}{\partial \eta}\right|_{\eta=0}=\lambda,\left.\quad \frac{\partial f}{\partial \eta}\right|_{n \rightarrow \infty}=1
$$

where $f(\eta, \xi)$ is the dimensionless stream function, $\alpha=$ $\alpha_{1} U / \rho x \nu$ is the local Deborah number, and ' denotes differentiation with respect to $\eta$. Notice that, for $\alpha=0$, the above equation reduces to that of the viscous fluid [9].

\section{HAM Solution}

To solve the problem (12) and (13) analytically, we use the well-known analytic technique homotopy analysis method. According to boundary conditions (13), it is convenient to express $f(\eta, \xi)$ by the base functions

$$
\left\{\xi^{k} \eta^{m} \exp (-n \eta) \mid k \geq 0, m \geq 0, n \geq 0\right\}
$$

in the following form:

$$
f(\eta, \xi)=\sum_{k=0}^{\infty} \sum_{m=0}^{\infty} \sum_{n=0}^{\infty} a_{m, n}^{k} \xi^{k} \eta^{m} \exp (-n \eta)
$$

where $a_{m, n}^{k}$ are coefficients involved in the solution series. According to the solution expression (15) and boundary conditions (13), we choose the initial approximation

$$
f_{0}(\eta, \xi)=\eta-\frac{1-\exp (-\beta \eta)}{\beta}+\lambda \eta \exp (-\beta \eta)
$$

and the linear operator

$$
\mathscr{L}[F(\eta, \xi ; p)]=\frac{\partial^{3} F}{\partial \eta^{3}}+\beta \frac{\partial^{2} F}{\partial \eta^{2}}
$$

satisfying the property

$$
\mathscr{L}\left[C_{1}+C_{2} \eta+C_{3} \exp (-\beta \eta)\right]=0
$$

where $C_{1}, C_{2}$, and $C_{3}$ are constants and $\beta$ is the controlling parameter to be adjusted. From (12), we define the nonlinear operator

$$
\begin{aligned}
\mathcal{N}[F(\eta, \xi ; p)] & \\
= & {[\xi-\alpha(1-\xi)] F^{\prime \prime \prime}(\eta, \xi ; p)-\xi^{2}(1-\xi) \frac{\partial F^{\prime}(\eta, \xi ; p)}{\partial \xi} } \\
& +\frac{1}{2} \eta \xi(1-\xi) F^{\prime \prime}(\eta, \xi ; p)+\frac{1}{2} \xi^{2} F(\eta, \xi ; p) F^{\prime \prime}(\eta, \xi ; p) \\
+\alpha & {\left[\xi(1-\xi) \frac{\partial F^{\prime \prime \prime}(\eta, \xi ; p)}{\partial \xi}-\frac{\eta}{2}(1-\xi) F^{i v}(\eta, \xi ; p)\right.} \\
& +\frac{\xi}{2} F^{\prime \prime 2}(\eta, \xi ; p)-\xi F^{\prime}(\eta, \xi ; p) F^{\prime \prime \prime}(\eta, \xi ; p) \\
& \left.-\frac{\xi}{2} F(\eta, \xi ; p) F^{i v}(\eta, \xi ; p)\right] .
\end{aligned}
$$

Taking $\hbar$ as nonzero auxiliary parameter, we construct the zero-order deformation equation [29]

$$
(1-p) \mathscr{L}\left[F(\eta, \xi ; p)-f_{0}(\eta)\right]=p \hbar \mathscr{N}[F(\eta, \xi ; p)]
$$

subject to the boundary conditions

$$
\begin{gathered}
F(0, \xi ; p)=0,\left.\quad \frac{\partial F(\eta, \xi ; p)}{\partial \eta}\right|_{\eta=0}=\lambda, \\
\left.\frac{\partial F(\eta, \xi ; p)}{\partial \eta}\right|_{\eta \rightarrow \infty}=1,
\end{gathered}
$$

where $p$ is the embedding parameter and obviously, when $p=$ 0 and $p=1,(20)$ has the solutions

$$
\begin{aligned}
& F(\eta, \xi ; 0)=f_{0}(\eta, \xi), \\
& F(\eta, \xi ; 1)=f(\eta, \xi),
\end{aligned}
$$

respectively. Thus, the variation of $p$ from 0 to 1 is continuous deformation of $f(\eta, \xi)$ from $f_{0}(\eta, \xi)$ to $f(\eta, \xi)$. Expanding $F(\eta, \xi ; p)$ in Taylor's series with respect to $p$, and using (22), we have

$$
F(\eta, \xi ; p)=f_{0}(\eta, \xi)+\sum_{m=1}^{\infty} f_{m}(\eta, \xi) p^{m}
$$

where

$$
f_{m}(\eta, \xi)=\left.\frac{1}{m !} \frac{\partial^{m} F(\eta, \xi ; p)}{\partial p^{m}}\right|_{p=0} .
$$

Assume that $\hbar$ is chosen so properly such that the series (24) is convergent at $p=1$. Using (22) and (23), we can write

$$
f(\eta, \xi)=f_{0}(\eta, \xi)+\sum_{m=1}^{\infty} f_{m}(\eta, \xi) .
$$


Differentiating the zero-order deformation equations (20) and (21) $m$-times with respect to $p$, at $p=0$ and then dividing by $m$ !, we obtain the $m$ th-order deformation equation

$$
\mathscr{L}\left[f_{m}(\eta, \xi)-\chi_{m} f_{m-1}(\eta, \xi)\right]=\hbar R_{m}(\eta, \xi)
$$

subject to the boundary conditions

$$
f_{m}(0, \xi)=0,\left.\quad \frac{\partial f_{m}}{\partial \eta}\right|_{\eta=0}=\left.\frac{\partial f_{m}}{\partial \eta}\right|_{\eta \rightarrow \infty}=0,
$$

where

$$
\begin{aligned}
& R_{m}(\eta, \xi) \\
& =[\xi-\alpha(1-\xi)] f_{m-1}^{\prime \prime \prime}-\frac{\alpha}{2} \eta(1-\xi) f_{m-1}^{i v} \\
& +\frac{1}{2} \eta \xi(1-\xi) f_{m-1}^{\prime \prime}+\alpha \xi(1-\xi) \frac{\partial f_{m-1}^{\prime \prime \prime}}{\partial \xi} \\
& -\xi^{2}(1-\xi) \frac{\partial f_{m-1}^{\prime}}{\partial \xi}+\frac{1}{2} \xi^{2} \sum_{k=0}^{m-1} f_{m-1-k} f_{k}^{\prime \prime} \\
& +\alpha\left[-\xi \sum_{k=0}^{m-1} f_{m-1-k}^{\prime} f_{k}^{\prime \prime \prime}-\frac{1}{2} \xi \sum_{k=0}^{m-1} f_{m-1-k} f_{k}^{i v}\right. \\
& \left.+\frac{1}{2} \xi \sum_{k=0}^{m-1} f_{m-1-k}^{\prime \prime} f_{k}^{\prime \prime}\right] \\
& \chi_{m}= \begin{cases}0, & m=1 \\
1, & m>1\end{cases}
\end{aligned}
$$

The system of linear nonhomogeneous equations (27)-(28) can be solved up to high order of approximation with the help of the symbolic computation software such as Mathematica.

Let $f_{m}^{*}(\eta, \xi)$ represent a special solution; then the general solution $f_{m}(\eta, \xi)$ can be determined as follows:

$$
f_{m}(\eta, \xi)=f_{m}^{*}(\eta, \xi)+C_{1}+C_{2} \eta+C_{3} e^{-\beta \eta},
$$

in which $C_{i}(i=1, \ldots, 3)$ are constants of integration which can be determined with the help of boundary conditions (28) as under

$$
C_{1}=-C_{3}-f_{m}^{*}(0, \xi), \quad C_{2}=0, \quad C_{3}=-\frac{1}{\beta} f_{m}^{* \prime}(0, \xi) \text {. }
$$

In this way, the complete solution for the present problem can be written in the form of an infinite series of functions; that is,

$$
f(\eta, \xi)=f_{0}(\eta, \xi)+\sum_{m=1}^{\infty} f_{m}(\eta, \xi)
$$

To prove that the series $f(\eta, \xi)$ is an approximate solution of the system (12) and (13), it is necessary to show the convergence of the solution series (32). As mentioned by Liao

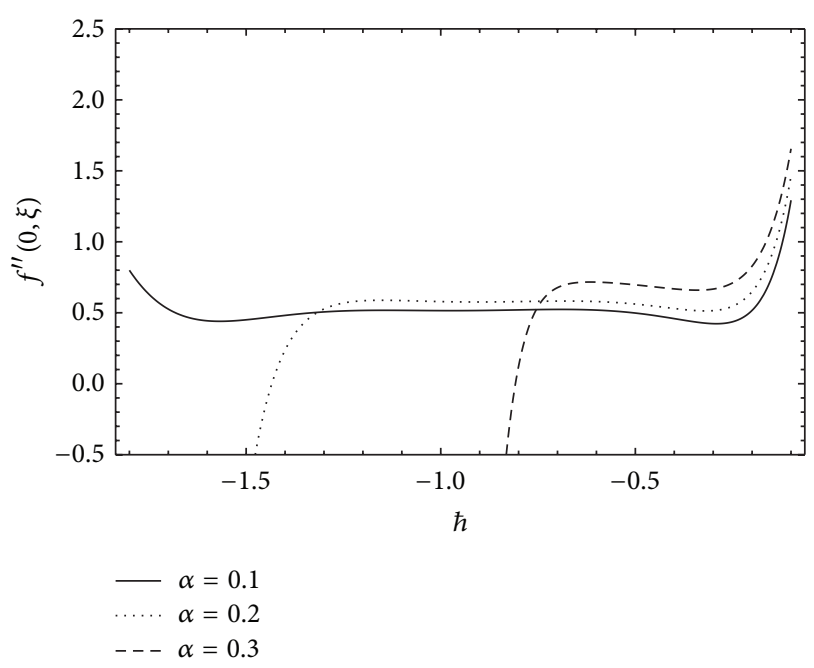

FIGURE 1 : $\hbar$-Curve for different values of the Deborah number $\alpha$ at the 19th order of approximation.

[29], the convergence of the solution series strongly depends upon the auxiliary parameter $\hbar$ once the initial guess and the linear operator have been selected. A rough estimate for the allowed regions of the values of $\hbar$, the so-called $\hbar$ curve, is important. We have plotted the $\hbar$-curve for our present problem in Figure 1. It is worth mentioning here that, in some problems with strong nonlinearity, the values of $\hbar$ strongly depend upon the involved physical parameters (see for instance [44]). In Figure 1, it can be seen that the intervals of allowed values of $\hbar$ are shrinking with increasing $\alpha$. Further, it can also be seen that, for higher values of $\alpha$, the interval of allowed values of $\hbar$ shifts towards zero. However, to search a more appropriate value of $\hbar$, it is useful to calculate the residual errors. In Figure 2, we have plotted the residual error against $\hbar$ for fixed values of the parameters involved. From Figure 2, it is clear that the error for $\alpha=0.1$ is minimum at $\hbar=-0.7736$ which is $4.257 \times 10^{-6}$ at the 20 th order of approximation. In Figure 3, we have plotted the errors graph against the space variable $\eta$ also. It shows that in the boundary layer region the error fluctuates and dies out to be zero as one moves to the outer region. Though the error fluctuates in the boundary layer region, it remains in the acceptable limits. In order to prove the convergence of the solution series, it is recommended that the corrections to solution must become negligible in the succeeding orders of approximation. We apply the homotopy Padé approximation in order to accelerate the convergence of solution series. In Table 1, we report the HAM solution at different orders of $[m, m]$ Padé approximation. Clearly, there are no corrections up to four decimal places after the 8th order of Padé approximation. This proves the convergence and accuracy of HAM solution.

\section{Graphical Illustration and Discussion of Results}

To understand the physics of flow phenomenon, we have investigated the solution through graphs. In Figure 4, the 


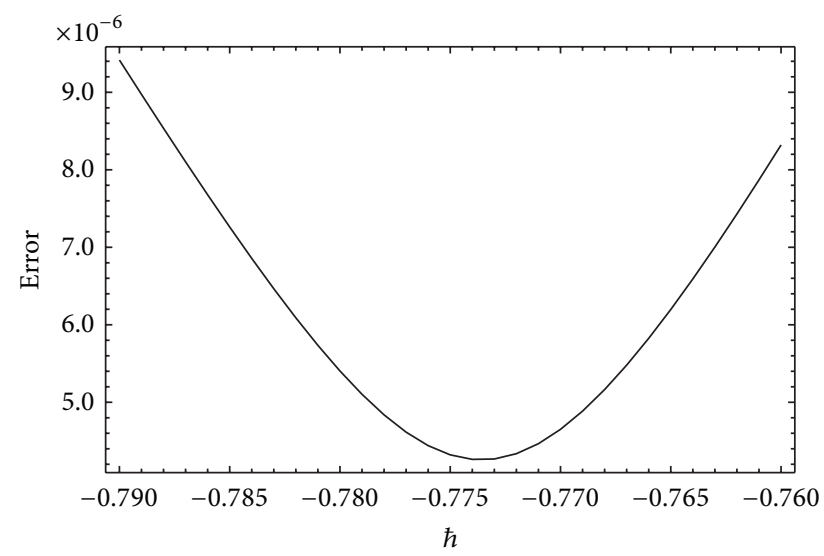

FIGURE 2: Squared relative error for the optimal values of $\hbar$ for $\alpha=$ 0.1 by keeping $\xi=0.8, \lambda=0$, and $\beta=5$ fixed.

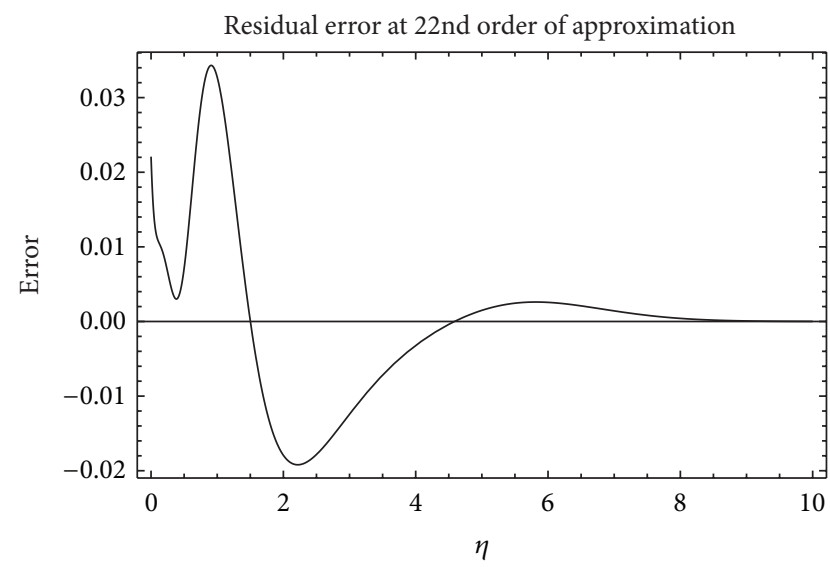

FIGURE 3: The residual error of the 22nd order of HAM solution in case of $\alpha=0.2, \xi=0.8, \lambda=0, \beta=5$, and $\hbar=-0.6815$.

TABLE 1: Convergence of HAM solution for different orders of padé approximation of $f^{\prime \prime}(0, \xi)$, when $\xi=0.8, \alpha=0.2, \beta=5$ and $\lambda=0$.

\begin{tabular}{lc}
\hline Order of approximation & $f^{\prime \prime}(0, \xi)$ \\
\hline$[2,2]$ & 0.4201 \\
{$[4,4]$} & 0.5460 \\
{$[6,6]$} & 0.5526 \\
{$[8,8]$} & 0.5650 \\
{$[10,10]$} & 0.5650 \\
{$[12,12]$} & 0.5650 \\
\hline
\end{tabular}

longitudinal component of velocity $f^{\prime}(\eta, \xi)$ is plotted for different values of the viscoelastic parameter $\alpha$. Clearly, strong non-Newtonian behavior of the fluid results in large skin friction at the solid wall. However, large values of $\alpha$ help in reducing the thickness of the boundary layer. In Figure 5, the velocity function $f^{\prime}(\eta, \xi)$ is plotted for different values of the parameter $\lambda$ (provided $\lambda \in(0,1)$ ). The velocity increases at the plate which causes the skin friction at the plate to reduce. This is due to the fact that, for $\lambda>0$, the plate and the free stream progress in the same direction, but, for $\lambda<0$ when the

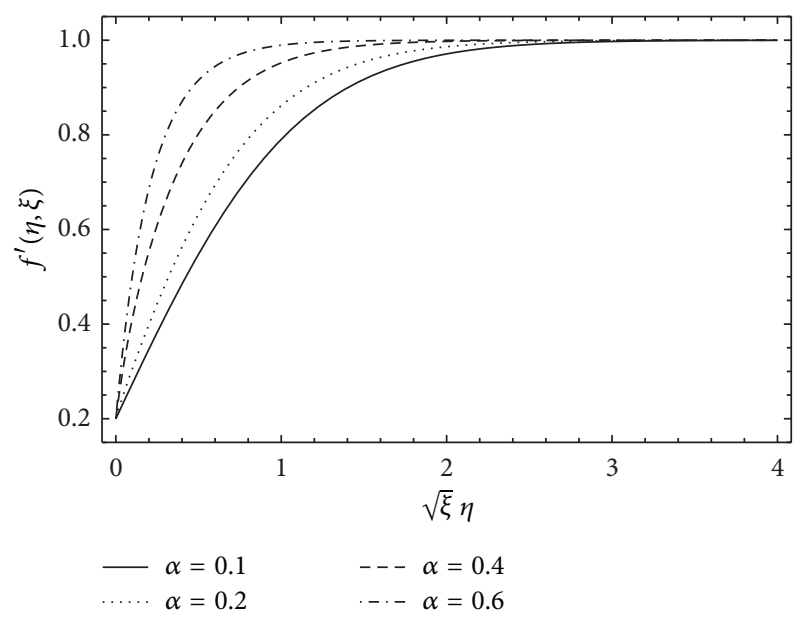

FIGURE 4: Effect of viscoelastic parameter $\alpha$ on the velocity profile when $\lambda=0.2, \xi=0.5$, and $\beta=5$ are kept fixed.

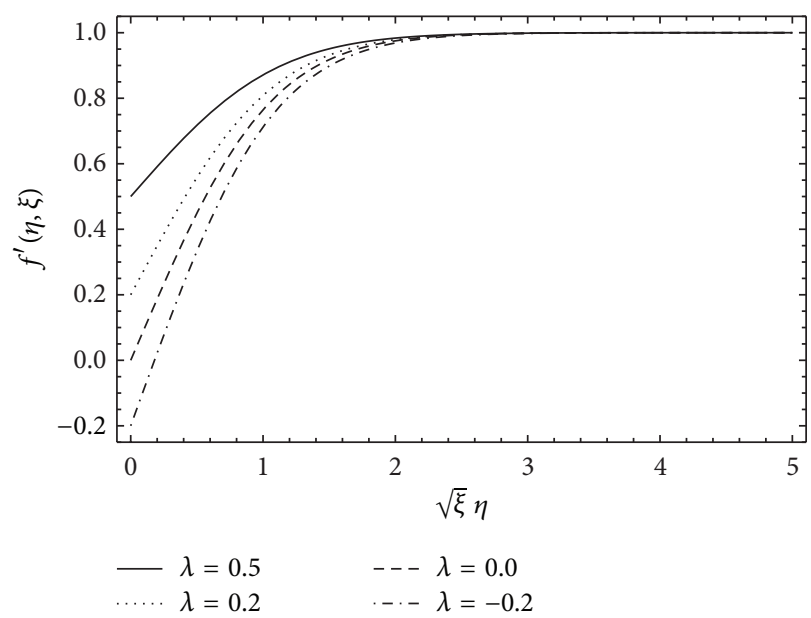

FIGURE 5: Effect of the parameter $\lambda$ on the velocity profile when $\alpha=$ $0.1, \xi=0.5$, and $\beta=5$ are kept fixed.

TABLE 2: Effect of viscoelastic parameter $\alpha$ on the boundary layer thickness $\delta$, when $\lambda=0.2, \beta=5$ and $\xi=0.5$.

\begin{tabular}{lc}
\hline$\alpha$ & Boundary layer thickness $\delta$ \\
\hline 0.00 & 2.2221 \\
0.01 & 2.2030 \\
0.05 & 2.3375 \\
0.10 & 2.4656 \\
0.20 & 2.1302 \\
0.40 & 1.5352 \\
0.60 & 1.0112 \\
0.80 & 0.9339 \\
1.00 & 0.8981 \\
1.20 & 0.8464 \\
\hline
\end{tabular}

plate and free stream have opposite direction of progression, the skin friction increases considerably. Finally, the present HAM solution is shown to be uniformly valid for all time 


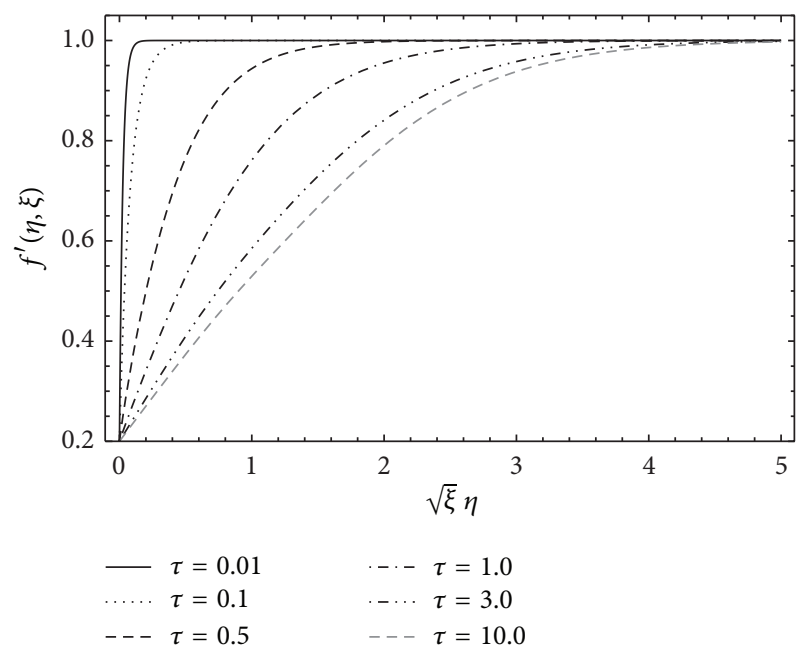

FIGURE 6: Velocity profile for different time $\tau$ when $\alpha=0.2, \lambda=0.2$, and $\beta=5$ are kept fixed.

$0 \leq \tau<\infty$ in the whole spatial domain $0 \leq \eta<\infty$ in Figure 6. Figure 6 depicts that the velocity is very small at initial time and with the passage of time the flow develops and reaches its steady state due to the motion of the plate. From the figure, it can also be observed that the steady state is reached at $\tau=5$ (roughly). This shows that the vorticity diffusion takes place in a very short interval of time.

The effect of viscoelastic parameter $\alpha$ on the boundary layer thickness $\delta$ is shown in Table 2. It is observed from the table that the thickness of the boundary layer, $\delta$, has a declining behavior with an increase in $\alpha$. For the low value of $\alpha$, this drop in $\delta$ is less as compared with the high value of $\alpha$. Such kind of result can be expected because at low values of $\alpha$ our governing equation behaves like that of Newtonian fluid [45]. At the low values of $\alpha$, shear stress produced in the flow is not so large so the boundary layer thickness increases. By increasing $\alpha$, the shear stress will grow more and more and results in the reduction of boundary layer thickness. We can observe such kind of results from the theory of polymeric liquid as well as experimental observations.

\section{Concluding Remarks}

In this study, we have considered the unsteady boundarylayer flow of a viscoelastic fluid over an impulsively started moving flat plate. Fluid at infinity was assumed to be flowing with a uniform free stream velocity. The governing nonlinear equations of an incompressible second-grade fluid are modelled using the similarity transformations. The resulting nonlinear problem is solved analytically with the help of HAM. The influence of various parameters of interest on the velocity profile is graphically illustrated. We find the following observations.

(i) It is noticed that the boundary layer thickness $\delta$ decreases by increasing second-grade parameter $\alpha$.

(ii) It is observed that as the time passes by, the unsteady velocity reaches the steady state showing that our analytic solution is valid for all the time throughout the spatial domain.

(iii) It is observed that increasing $\lambda$ (for $\lambda \in(0,1))$ reduces the drag at the plate whereas for $\lambda<0$ the skin friction at the plate increases by increasing $|\lambda|$.

\section{Conflict of Interests}

The authors declare that they have no conflict of interests regarding the publication of this paper.

\section{References}

[1] L. Prandtl, "Über Flüssigkeitsbewegung bei sehr kleiner Reibung," in Verhandlungen des 3rd internationalen MathematikerKongresses, pp. 484-491, Teubner, Heidelberg, Germany, 1904.

[2] H. Blasius, "Grenzschiechten in flüssigkeiten mit kleiner reibung," Zeitschrift für Angewandte Mathematik und Physik, vol. 56, pp. 1-37, 1908.

[3] B. Sakiadis, "Boundary-layer behavior on continuous solid surface: II. The boundary layer on a continuous flat surface," AIChE Journal, vol. 7, no. 2, pp. 221-225, 1961.

[4] F. K. Tsou, E. M. Sparrow, and R. J. Goldstein, "Flow and heat transfer in the boundary layer on a continuous moving surface," International Journal of Heat and Mass Transfer, vol. 10, no. 2, pp. 219-235, 1967.

[5] J. P. Klemp and A. Acrivos, "A method for integrating the boundary layer equations through a region of reverse flow," Journal of Fluid Mechanics, vol. 53, no. 1, pp. 177-191, 1972.

[6] M. Y. Hussaini, W. D. Lakin, and A. Nachman, "On similarity solutions of a boundary layer problem with an upstream moving wall," SIAM Journal on Applied Mathematics, vol. 47, no. 4, pp. 699-709, 1987.

[7] T. Fang, "Similarity solutions for a moving-flat plate thermal boundary layer," Acta Mechanica, vol. 163, no. 3-4, pp. 161-172, 2003.

[8] T. Fang, "Further study on a moving-wall boundary-layer problem with mass transfer," Acta Mechanica, vol. 163, no. 3-4, pp. 183-188, 2003.

[9] A. Mehmood and A. Ali, "Unsteady boundary layer flow due to an impulsively started moving plate," Proceedings of the Institution of Mechanical Engineers G: Journal of Aerospace Engineering, vol. 221, no. 3, pp. 385-390, 2007.

[10] A. Mehmood, A. Ali, and T. Shah, "Unsteady boundarylayer viscous flow due to an impulsively started porous plate," Canadian Journal of Physics, vol. 86, no. 9, pp. 1079-1082, 2008.

[11] E. G. Fisher, Extrusion of Plastics, Wiley, New York, NY, USA, 1976.

[12] Z. Tadmor and I. Klein, Engineering Principles of Plastication Extrusion, Polymer Science and Engineering Series, D. Van Nostrand Reinhold, New York, NY, USA, 1970.

[13] V. G. Fox, L. E. Erickson, and L. T. Fan, "The laminar boundary layer on a moving continuous flat sheet immersed in a nonNewtonian fluid," AIChE Journal, vol. 15, no. 3, pp. 327-333, 1969.

[14] I. A. Hassanien, "Flow and heat transfer on a continuous flat surface moving in a parallel free stream of power-law fluid," Applied Mathematical Modelling, vol. 20, no. 10, pp. 779-784, 1996. 
[15] I. A. Hassanien, "Flow and heat transfer from a continuous surface in a parallel free stream of viscoelastic second-order fluid," Applied Scientific Research, vol. 49, no. 4, pp. 335-344, 1992.

[16] S. C. R. Dennis, "The motion of a viscous fluid past an impulsively started semi-infinite flat plate," IMA Journal of Applied Mathematics, vol. 10, no. 1, pp. 105-117, 1972.

[17] R. Seshadri, N. Sreeshylan, and G. Nath, "Unsteady mixed convection flow in the stagnation region of a heated vertical plate due to impulsive motion," International Journal of Heat and Mass Transfer, vol. 45, no. 6, pp. 1345-1352, 2002.

[18] R. Nazar, N. Amin, and I. Pop, "Unsteady boundary layer flow due to a stretching surface in a rotating fluid," Mechanics Research Communications, vol. 31, no. 1, pp. 121-128, 2004.

[19] S. J. Liao, "An analytic solution of unsteady boundary-layer flows caused by an impulsively stretching plate," Communications in Nonlinear Science and Numerical Simulation, vol. 11, no. 3, pp. 326-339, 2006.

[20] H. Xu and S. J. Liao, "Series solutions of unsteady magnetohydrodynamic flows of non-Newtonian fluids caused by an impulsively stretching plate," Journal of Non-Newtonian Fluid Mechanics, vol. 129, no. 1, pp. 46-55, 2005.

[21] H. Xu, S. J. Liao, and I. Pop, "Series solutions of unsteady boundary layer flow of a micropolar fluid near the forward stagnation point of a plane surface," Acta Mechanica, vol. 184, no. 1-4, pp. 87-101, 2006.

[22] J. Cheng, S. J. Liao, and I. Pop, "Analytic series solution for unsteady mixed convection boundary layer flow near the stagnation point on a vertical surface in a porous medium," Transport in Porous Media, vol. 61, no. 3, pp. 365-379, 2005.

[23] C. Wang, "Analytic solutions for a liquid film on an unsteady stretching surface," Heat and Mass Transfer, vol. 42, no. 8, pp. 759-766, 2006.

[24] S. Abbasbandy, E. Babolian, and M. Ashtiani, "Numerical solution of the generalized Zakharov equation by homotopy analysis method," Communications in Nonlinear Science and Numerical Simulation, vol. 14, no. 12, pp. 4114-4121, 2009.

[25] H. Xu and S. J. Liao, "Series solutions of unsteady magnetohydrodynamic flows of non-Newtonian fluids caused by an impulsively stretching plate," Journal of Non-Newtonian Fluid Mechanics, vol. 129, no. 1, pp. 46-55, 2005.

[26] A. Mehmood and A. Ali, "Analytic homotopy solution of generalized three-dimensional channel flow due to uniform stretching of the plate," Acta Mechanica Sinica, vol. 23, no. 5, pp. 503-510, 2007.

[27] A. Mehmood, A. Ali, and T. Shah, "Heat transfer analysis of unsteady boundary layer flow by homotopy analysis method," Communications in Nonlinear Science and Numerical Simulation, vol. 13, no. 5, pp. 902-912, 2008.

[28] S. Munawar, A. Mehmood, and A. Ali, "Time-dependent flow and heat transfer over a stretching cylinder," Chinese Journal of Physics, vol. 50, no. 5, pp. 828-848, 2012.

[29] S. J. Liao, Beyond Perturbation: Introduction to Homotopy Analysis Method, Chapman \& Hall/CRC, Boca Raton, Fla, USA, 2004.

[30] S. Abbasbandy, "Homotopy analysis method for heat radiation equations," International Communications in Heat and Mass Transfer, vol. 34, no. 3, pp. 380-387, 2007.

[31] S. Abbasbandy and F. S. Zakaria, "Soliton solutions for the fifth-order KdV equation with the homotopy analysis method," Nonlinear Dynamics, vol. 51, no. 1-2, pp. 83-87, 2008.
[32] C. Wang, J. M. Zhu, S. J. Liao, and I. Pop, "On the explicit analytic solution of Cheng-Chang equation," International Journal of Heat and Mass Transfer, vol. 46, no. 10, pp. 1855-1860, 2003.

[33] H. Xu, "An explicit analytic solution for free convection about a vertical flat plate embedded in a porous medium by means of homotopy analysis method," Applied Mathematics and Computation, vol. 158, no. 2, pp. 433-443, 2004.

[34] C. Yang and S. Liao, "On the explicit, purely analytic solution of von Kármán swirling viscous flow," Communications in Nonlinear Science and Numerical Simulation, vol. 11, no. 1, pp. 83-93, 2006.

[35] M. Zakaria, "Magnetohydrodynamic viscoelastic boundary layer flow past a stretching plate and heat transfer," Applied Mathematics and Computation, vol. 155, no. 1, pp. 165-177, 2004.

[36] S. Munawar, A. Mehmood, and A. Ali, "Effects of slip on flow between two stretchable disks using optimal homotopy analysis method," Canadian Journal of Applied Sciences, vol. 1, pp. 50-68, 2011.

[37] F. M. Allan and M. I. Syam, "On the analytic solutions of the nonhomogeneous Blasius problem," Journal of Computational and Applied Mathematics, vol. 182, no. 2, pp. 362-371, 2005.

[38] A. Mehmood, S. Munawar, and A. Ali, "Cooling of a hot stretching surface in the presence of across mass transfer phenomenon in a channel flow," Zeitschrift für Naturforschung A-A Journal of Physical Sciences, vol. 69, pp. 34-42, 2014.

[39] A. Mehmood and A. Ali, "Analytic solution of threedimensional viscous flow and heat transfer over a stretching flat surface by homotopy analysis method," Journal of Heat Transfer, vol. 130, no. 12, Article ID 121701, 2008.

[40] S. J. Liao, "A new branch of solutions of boundary-layer flows over an impermeable stretched plate," International Journal of Heat and Mass Transfer, vol. 48, no. 12, pp. 2529-2539, 2005.

[41] A. S. Butt, A. Ali, and S. Munawar, "Slip effects on entropy generation in MHD flow over a stretching surface in the presence of thermal radiation," International Journal of Exergy, vol. 13, no. 1, pp. 1-20, 2013.

[42] R. S. Rivlin and J. L. Ericksen, "Stress-deformation relations for isotropic materials," Journal of Rational Mechanics and Analysis, vol. 4, no. 2, pp. 323-425, 1955.

[43] R. L. Fosdick and K. R. Rajagopal, "Anomalous features in the model of 'second order fluids"' Archive for Rational Mechanics and Analysis, vol. 70, no. 2, pp. 145-152, 1979.

[44] A. Mehmood and A. Ali, "An explicit analytic solution of steady three-dimensional stagnation point flow of second grade fluid toward a heated plate," Journal of Applied Mechanics, vol. 75, no. 6, Article ID 061003, 2008.

[45] R. B. Bird, R. C. Armstrong, and O. Hassager, Dynamics of Polymeric Liquids, vol. 1, Wiley, New York, NY, USA, 2nd edition, 1987. 

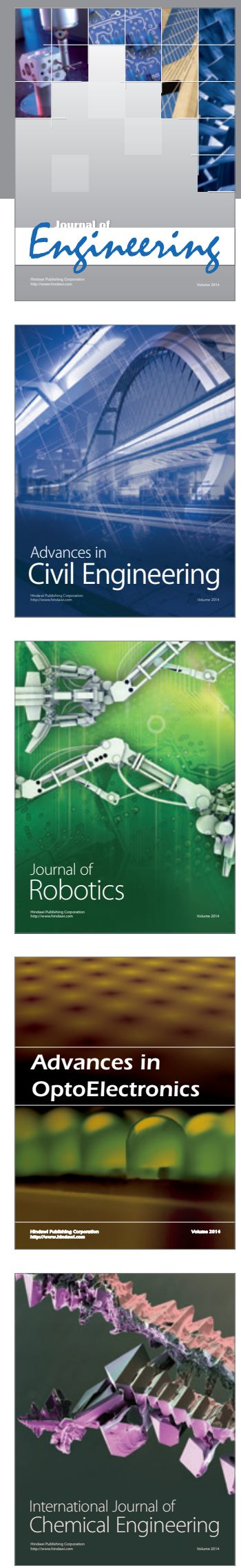

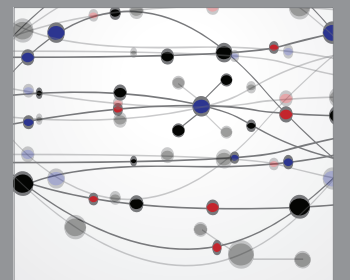

The Scientific World Journal
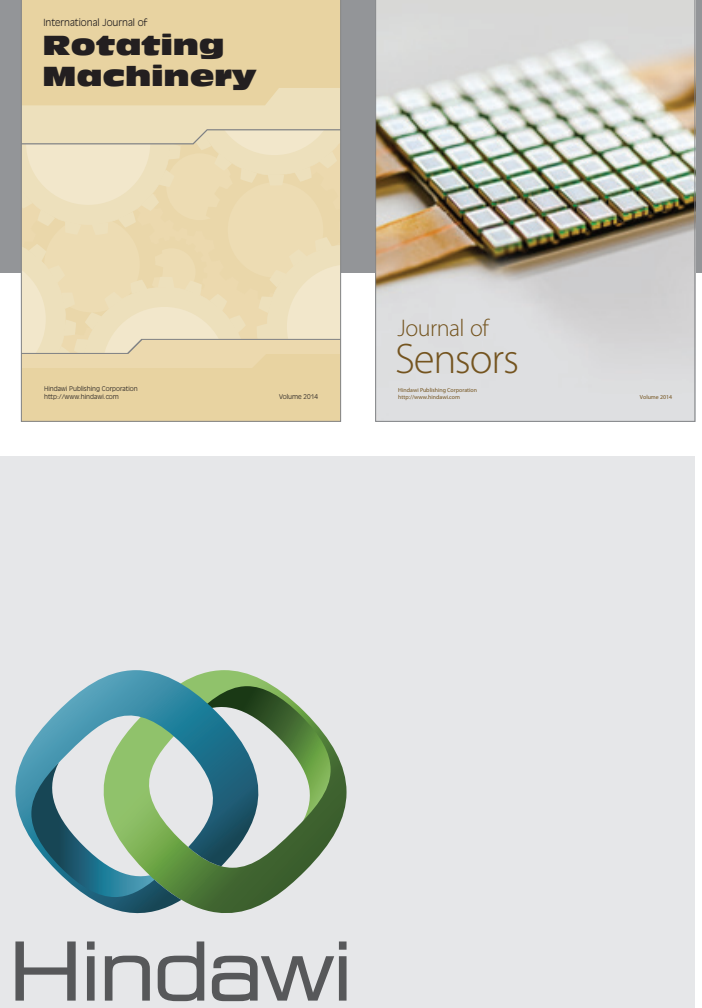

Submit your manuscripts at http://www.hindawi.com
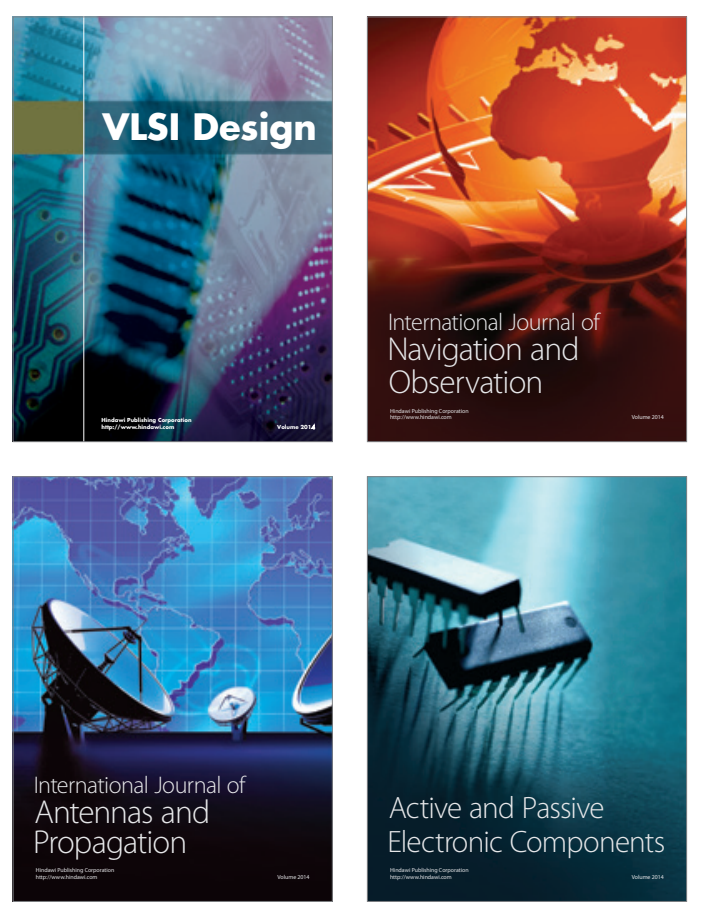
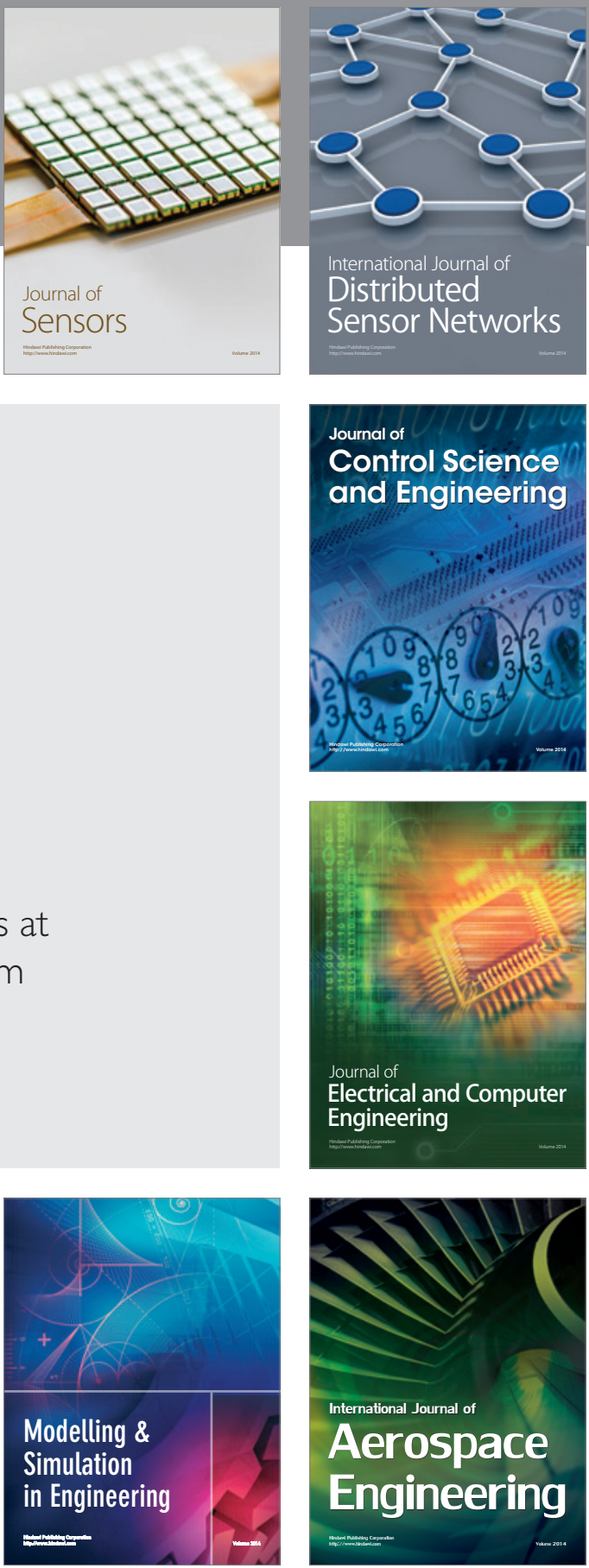

Journal of

Control Science

and Engineering
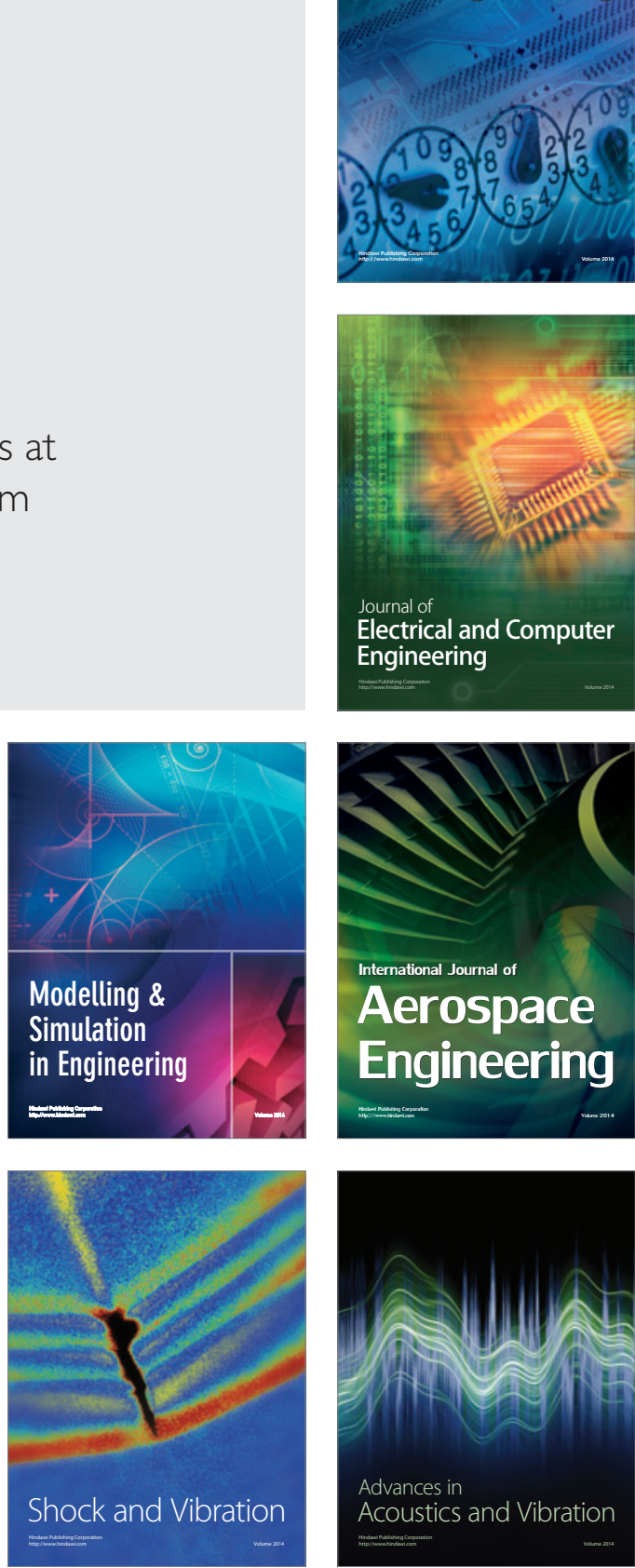